
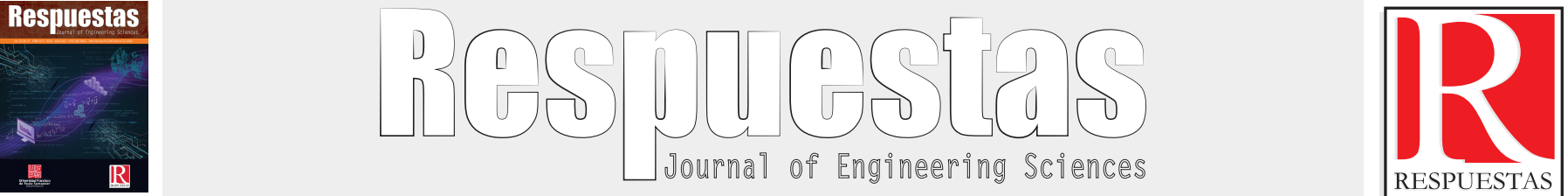

Artículo Original

https://doi.org/10.22463/0122820X.1493

\title{
Análisis de vitamina B2 en arroz blanco comercializado en el Área Metropolitana de Cúcuta por cromatografía líquida ultra rápida (UFLC)
}

Analysis of vitamin B2 in white rice commercialized in the Metropolitan Area of Cúcuta by ultra-fast liquid chromatography (UFLC)

Dora Cecilia Rodríguez-Ordoñez, Laura Natalia Torres-Herrera.

${ }_{b}^{a}$ Magister en Quimica, doraceciliaro@ufps.edu.co, orcid.org/0000-0002-8037-9799 Universidad Francisco de Paula Santander, Cúcuta, Colombia.

Tecnóloga Quimica, lauranataliath@ufps.edu.co, orcid.org/0000-0001-6915-5316 Universidad Francisco de Paula Santander, Cúcuta, Colombia.

Cómo citar: D.C. Rodríguez-Ordoñez y L. N. Torres-Herrera, "Análisis de vitamina B2 en arroz blanco comercializado en el Área Metropolitana de

Cúcuta por cromatografía líquida ultra rápida (UFLC)", Respuestas, vol. 23, no. S1, pp. 19-23, 2018. https://doi.org/10.22463/0122820X.1493

Recibido: Febrero 9 de 2018; Aprobado: Julio 27 de 2018.

\begin{tabular}{|c|c|}
\hline & RESUMEN \\
\hline $\begin{array}{l}\text { Palabra Clave: } \\
\text { Cereales } \\
\text { Fluorescencia } \\
\text { HPLC } \\
\text { Riboflavina } \\
\text { Vitaminas }\end{array}$ & $\begin{array}{l}\text { Antecedente: el análisis de riboflavina es uno de los parámetros mayormente referenciado en la determinación del valor nutritivo } \\
\text { de algunos alimentos. Objetivo: estandarizar el método de análisis y determinar el contenido de riboflavina (vitamina B2) en } \\
\text { muestras de arroz blanco fortificado (A y B) y sin fortificar (C y D), comercializadas en el área metropolitana de Cúcuta. } \\
\text { Métodos: la extracción y análisis de dos lotes de cada muestra se realizó por triplicado. Las muestras sin fortificar se doparon } \\
\text { con el fin de determinar el porcentaje de recuperación del método. Tres muestras de } 5,0000 \text { gramos de cada lote de arroz } \\
\text { fortificado y sin fortificar, fueron sometidas al proceso de extracción de la vitamina mediante hidrólisis ácida, previo tratamiento } \\
\text { enzimático con papána y takadiastasa a } 45^{\circ} \mathrm{C} \text {. Los extractos se purificaron filtrando por gravedad con papel filtro, y nuevamente } \\
\text { por filtros de jeringa de celulosa regenerada de } 0,20 \mu \mathrm{m} \text {. Se determinó por triplicado la concentración de riboflavina en cada } \\
\text { extracto, mediante HPLC en un cromatógrafo líquido UFLC SHIMADZU Prominence. El análisis estadístico de los datos se } \\
\text { realizó con el software R versión } 3.2 .2 \text {. Resultados: las concentraciones promedio, en mg de riboflavina/kg de arroz, en las } \\
\text { muestras de arroz A, B, C y D fueron de } 0,4121,2,3741,0,3208 \text { y } 0,3217 \text {, respectivamente para el lote } 1 \text { y para el lote } 2 \text { de } \\
2,3831,1,0552,0,4022, \text { y } 0,3246 \text {, respectivamente. Los porcentajes de recuperación de riboflavina en las muestras dopadas } \\
\text { estuvieron alrededor del } 91,00 \% \text {. Conclusiones: se observa que entre diferentes lotes de arroz fortificado de la misma marca se } \\
\text { presenta variación en el contenido de la vitamina. En las muestras de arroz no fortificado, la concentración hallada corresponde } \\
\text { al contenido natural de riboflavina en el arroz, con valores muy similares en todas las muestras. }\end{array}$ \\
\hline
\end{tabular}

\section{ABSTRACT}

\section{Keywords:}

Cereals

Fluorescence

HPLC

Riboflavin

Vitamins
Background: Riboflavin analysis is one of the parameters most referenced in the determination of the nutritional value of some foods. Objective: standardize the method of analysis and determine the content of riboflavin (vitamin B2) in samples of white rice fortified ( $\mathrm{A}$ and $\mathrm{B})$ and unfortified $(\mathrm{C}$ and $\mathrm{D})$, marketed in the metropolitan area of Cúcuta. Method: the extraction and triplicate analysis of two batches of each sample was performed. Unfortified samples were doped in order to determine the percent recovery of the method. Three samples of 5,0000 grams of each batch of fortified and non-fortified rice, were subjected to the extraction process of the vitamin by acid hydrolysis, after enzymatic treatment with papain and takadiastase at $45^{\circ} \mathrm{C}$. The extracts were purified by filtering with filter paper, and again by regenerated cellulose syringe filters of $0.20 \mu \mathrm{m}$. The riboflavin concentration in each extract was determined in triplicate by HPLC on a UFLC SHIMADZU Prominence liquid chromatograph. The statistical analysis of data was carried out with the software $\mathrm{R}$ version 3.2.2. Results: the average concentrations, in $\mathrm{mg}$ riboflavin / $\mathrm{kg}$ of rice, in rice samples A, B, C and D were $0,4121,2,3741,0,3208$ and 0,3217 , respectively for batch 1 , and for batch 2 were 2,3831, 1,0552, 0,4022 and 0,3246, respectively. Recovery percentages of riboflavin in doped samples were about $91.00 \%$. Conclusion: it is noted that between different batches of fortified rice of the same brand there is variation in the content of vitamin. In samples of not fortified rice, the concentration found corresponds to the natural riboflavin content in rice, with similar values in all samples.

\section{Introducción}

Las vitaminas son esenciales en el metabolismo, se encuentran en pequeñas cantidades en los alimentos y son necesarias para que el organismo funcione correctamente [1], actúan generalmente como catalizadores al combinarse con las proteínas creando metabolitos de enzimas activas, produciendo reacciones químicas muy importantes en el organismo [2].

Las vitaminas se clasifican en dos grandes grupos, hidrosolubles y liposolubles, característica que define la reacción de asimilación que se llevará a cabo en el organismo. Dentro de las hidrosolubles se encuentran las vitaminas del complejo B formado por tiamina, riboflavina, niacina, piridoxina, ácido pantoténico, biotina, cobalamina y ácido fólico [3]. La riboflavina (vitamina B2), se encuentra generalmente fosforilada integrando las coenzimas; por lo que debe asociarse a una porción de otra enzima para catalizar diversos procesos de oxidación-reducción [4]. Estudios recientes han proporcionado evidencia de que la vitamina B2, actúa como un sensibilizante para mejorar la eficacia de la quimioprevención y la quimioterapia del cáncer a base de vitamina $\mathrm{C}$, debido a que la combinación de las dos vitaminas puede lograr una actividad anticancerígena sinérgica [5].

*Autor de correspondencia.

E-mail: doraceciliaro@ufps.edu.co (Dora Cecilia Rodríguez Ordoñez),

cc) (i) (s) $\odot$ Peer review is the responsibility of the Universidad Francisco de Paula Santander. cc) (j) $(-)$ This is an article under the license CC BY-NC-ND 
El arroz, pasa por procesos de refinado y pulido que provocan la pérdida casi total de varios de sus nutrientes, entre estos las vitaminas [6],[7], en algunos casos pierde hasta un $85 \%$ de ellas. Por esto el arroz al igual que otros cereales, que carecen de vitaminas A, D y C, pero que contienen pequeñas cantidades de tiamina, riboflavina y niacina, son candidatos a procesos de fortificación con vitaminas para elevar su valor nutricional [8].

Para el análisis de la riboflavina se utilizan métodos fluorométricos, microbiológicos y espectrofotométricos, sin embargo, la cromatografía liquida de alta resolución (High Performance Liquid Chromatography - HPLC) es una técnica que minimiza los tiempos de análisis con alta sensibilidad y especificidad, en comparación con los mencionados [9].

Entre los trabajos que reportan el análisis de vitamina B2 en alimentos mediante HPLC se encuentran, la determinación de riboflavina en muestras de carne e hígado fresco [10], Arthrospira máxima [11], jarabes infantiles en el cual comparan dos técnicas de análisis (fluorometría y HPLC) [12], en harinas de lenteja [13], productos lácteos [14], harina de trigo [15] y en leche en polvo comercial y en tabletas [16].

En este trabajo se estandarizó el método de análisis de riboflavina, el cual se utilizó para la cuantificación de la vitamina presente en arroz blanco fortificado y sin fortificar, el tratamiento estadístico de los datos obtenidos en el análisis de cuatro marcas comerciales de arroz, por cromatografía liquida ultra rápida (Ultra Fast Liquid Chromatography-UFLC) con detección de fluorescencia, se llevó a cabo con el software $R$ versión 3.2.2 [17]. Las muestras fortificadas fueron distribuidas por arroceras del Norte de Santander y las no fortificadas por arroceras nacionales.

\section{Materiales y métodos}

\section{Estandarización del método}

La estandarización del método para análisis de vitamina B2 en arroz blanco se basó en el procedimiento descrito por el Instituto de Salud Pública de Chile (ISP) [18]. A partir de un estándar de riboflavina (SIGMA ALDRICH), se elaboró la curva de calibración con patrones entre 0,1000 y 1,500 ppm, y con el fin de determinar el límite de detección y de cuantificación se prepararon patrones de concentración desde $2,500 \times 10^{-4}$ hasta $5,000 \times 10^{-2}$ ppm de riboflavina [19].

\section{Selección y Preparación de las muestras}

Selección de las muestras: las muestras se seleccionaron según criterio de fortificación, es decir, fortificadas con riboflavina (A y B) y no fortificadas (C y D), de manera aleatoria en dos tiendas de cadena del área metropolitana de San José de Cúcuta (Norte de Santander). Las muestras comerciales de arroz que se adquirieron para el análisis fueron de dos lotes de producción con fecha de distribución mayor a un mes, con el fin de garantizar la diferencia entre muestras de la misma marca, para su posterior comparación. Las muestras fortificadas (A y B) corresponden a marcas registradas por dos arroceras con domicilio en el área metropolitana de San José de Cúcuta y las no fortificadas (C y D) son distribuidas por dos empresas que cuentan con molinos arroceros a nivel nacional.

Preparación de las muestras: se tomó por triplicado 5,0000 gramos de cada muestra molida, tamizada y homogenizada. A cada muestra se le adicionó 0,5000 gramos de takadiastasa (SIGMA ALDRICH), 0,2500 gramos de papaína (Merck) y 15 $\mathrm{mL}$ de agua desionizada a $45^{\circ} \mathrm{C}$, se mantuvo la temperatura en baño maría con agitación por 30 minutos para realizar el tratamiento enzimático. Posteriormente se dio inicio a la hidrólisis ácida, para esto se agregaron $30 \mathrm{~mL}$ de agua desionizada a $45^{\circ} \mathrm{C}$ y 5,0 mL de solución de ácido clorhídrico $(\mathrm{HCl}) 1,000 \mathrm{M}$, llevando a calentamiento y agitación en baño maría por 30 minutos más. Finalizado el tiempo de hidrólisis, a la muestra a temperatura ambiente, se le adicionaron $5,0 \mathrm{~mL}$ de solución de acetato de sodio $\left(\mathrm{CH}_{3} \mathrm{COONa}\right) 2,500 \mathrm{M}$. La muestra se transfirió a un balón ámbar y aforó a 100,0 mL, luego se filtró por gravedad y el filtrado se purificó pasándolo por membranas de celulosa regenerada (marca Sartorius) de $15 \mathrm{~mm}$ y 0,20 $\mu \mathrm{m}$. Con el fin de determinar el porcentaje de recuperación del método, fueron dopadas muestras de las marcas no fortificadas (C y D) con 5,000 ppm de estándar de riboflavina, posteriormente fueron sometidas al proceso de extracción y cuantificación. Cabe resaltar que debido a que la riboflavina es sensible a la luz, fue necesario realizar todo el procedimiento de preparación en condiciones de luz controlada [18].

\section{Análisis de patrones y muestras}

Los patrones de calibración y los extractos de las muestras de arroz blanco se llevaron a viales ámbar de 1,5 mL, para su análisis en un equipo UFLC SHIMADZU serie Prominence, con automuestreador SIL-20AHT, calefactor de columna CTO-20A, detector de fluorescencia RF-20A y módulo de control CBM-20A, provisto del software LABSOLUTIONS de SHIMADZU. El análisis se realizó utilizando N,N-dimetilformamida al $30 \%$ en solución de hidrogenofosfasto de dipotasio a pH 7,20 como fase móvil, con caudal de $0,8 \mathrm{~mL} / \mathrm{min}$ a elución isocrática en fase reversa, utilizando columna C18 de 4,6 x 50 mm y precolumna C18 marca SHIMADZU. La detección en el detector RF-20A se realizó a una longitud de excitación de 450 $\mathrm{nm}$ y de emisión de $528 \mathrm{~nm}$ [18].

Establecidas las condiciones cromatográficas para el método, se corrieron tanto los patrones de calibración, como los extractos de las muestras, inyectando por triplicado $20 \mu \mathrm{L}$ de cada una de las soluciones.

\section{Análisis de los datos}

Se analizó la precisión de los datos obtenidos en la estandarización del método y en el análisis de las muestras, mediante la 
determinación de la desviación estándar y el coeficiente de variación. Los datos de concentración (mg de riboflavina/ $\mathrm{kg}$ de arroz) obtenidos en el análisis de las muestras de cada uno de los lotes, denominadas A1, A2, B1, B2, C1, C2, D1 y D2, se analizaron con el software $R$ versión 3.2.2., mediante el cual se realizó la comparación de la concentración de riboflavina presente en todas las muestras y por separado se comparó la concentración entre lotes de la misma marca, con el fin de observar si se presentaba o no, diferencia significativa en el contenido de vitamina, teniendo en cuenta que para valores de probabilidad $(p)>0,05$, se considera que no existe diferencia significativa entre los datos.

\section{Resultados y análisis}

\section{Estandarización del método}

El procedimiento del ISP de Chile [18] reporta un intervalo lineal entre 0,1000 y $1,000 \mathrm{ppm}$ de riboflavina, sin embargo, al analizar los patrones de menor concentración para determinar el límite de detección y de cuantificación del método se estableció que existe linealidad para el análisis de riboflavina desde $2,500 \times 10^{-4}$ hasta $1,500 \mathrm{ppm}$, con un coeficiente de correlación de 0,99997, como se muestra en la Figura 1, y al extrapolar a cero se mantiene el mismo coeficiente de correlación. La precisión en las mediciones de los patrones de calibración por UFLC, se evidenció con valores de coeficientes de variación $(\% \mathrm{CV}$ ) bajos (entre el 0,052 y el 5,262\%), como se muestra en la Tabla I.

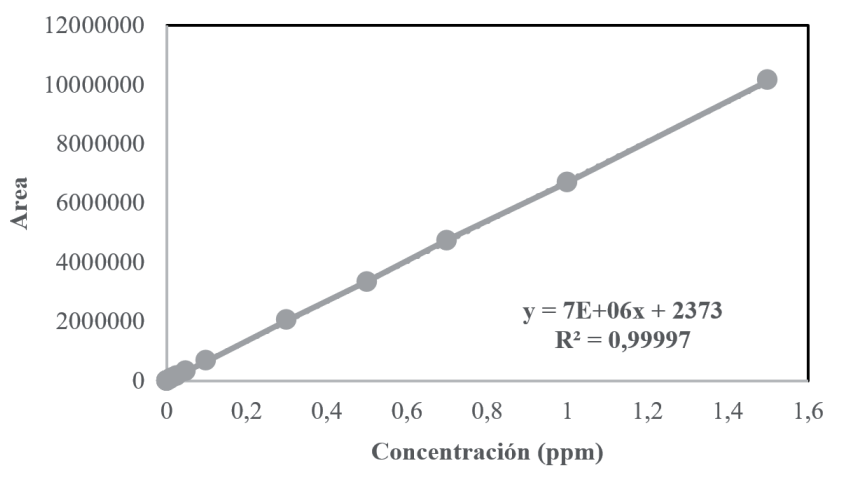

Figura 1. Curva de calibración para análisis de riboflavina por UFLC

Tabla I. Datos para elaboración de la curva de calibración de riboflavina por UFLC

\begin{tabular}{|c|c|c|c|}
\hline AREA PROMEDIO & $\begin{array}{c}\text { CONCENTRACION } \\
\text { (mg de vitamina B2/kg de arroz) }\end{array}$ & DESVEST & \% CV \\
\hline 3349,0 & $\mathbf{2 , 5 0 0 \times 1 0 ^ { - 4 }}$ & 23,07 & 0,689 \\
\hline 6754,0 & $\mathbf{5 , 5 0 0 \times 1 0 ^ { - 4 }}$ & 223,42 & 3,308 \\
\hline 10362,3 & $\mathbf{1 , 1 0 0 \times 1 0 ^ { - 3 }}$ & 231,37 & 2,233 \\
\hline 17898,3 & $\mathbf{2 , 2 0 0 \times 1 0 ^ { - 3 }}$ & 941,88 & 5,262 \\
\hline 45740,3 & $\mathbf{6 , 5 0 0 \times 1 0 ^ { - 3 }}$ & 889,87 & 1,945 \\
\hline 86841,7 & $\mathbf{0 , 0 1 2 5 0}$ & 508,81 & 0,586 \\
\hline 171788,3 & $\mathbf{0 , 0 2 5 0 0}$ & 1148,73 & 0,669 \\
\hline 337121,3 & $\mathbf{0 , 0 5 0 0 0}$ & 256,47 & 0,076 \\
\hline 674454,0 & $\mathbf{0 , 1 0 0 0}$ & 8649,78 & 1,282 \\
\hline 2047061,0 & $\mathbf{0 , 3 0 0 0}$ & 5764,46 & 0,282 \\
\hline 3351190,7 & $\mathbf{0 , 5 0 0 0}$ & 6367,57 & 0,190 \\
\hline 4751084,0 & $\mathbf{0 , 7 0 0 0}$ & 3169,26 & 0,067 \\
\hline 6701046,7 & $\mathbf{1 , 0 0 0 0}$ & 6158,73 & 0,092 \\
\hline 10136805,7 & $\mathbf{1 , 5 0 0 0}$ & 5317,58 & 0,052 \\
\hline
\end{tabular}

\section{Análisis de las muestras}

Con la ecuación obtenida para la curva de calibración del método (véase Figura 1), se calculó la concentración de riboflavina en cada una de las muestras, expresada en mg de riboflavina/kg de arroz, los valores promedio, la desviación estándar (desvest) y los coeficientes de variación $(\% \mathrm{CV})$ obtenidos para cada una de las marcas se muestran en la Tabla II.

Tabla II. Valores promedio de la concentración de riboflavina en las marcas comerciales de arroz electrónica

\begin{tabular}{|c|c|c|c|c|}
\hline $\begin{array}{c}\text { Marca } \\
\text { de arroz }\end{array}$ & Lote & $\begin{array}{c}\text { CONCENTRACION } \\
\text { (mg de vitamina B2/kg de arroz) }\end{array}$ & DESVEST & \% CV \\
\hline \multirow{2}{*}{$\mathrm{A}$} & $\mathrm{A} 1$ & 0,4121 & 0,0294 & 7,1330 \\
\cline { 2 - 5 } & $\mathrm{A} 2$ & 2,3831 & 0,1885 & 7,9111 \\
\hline \multirow{2}{*}{$\mathrm{B}$} & $\mathrm{B} 1$ & 2,3741 & 0,1963 & 8,2669 \\
\cline { 2 - 5 } & $\mathrm{B} 2$ & 1,0552 & 0,0515 & 4,8779 \\
\hline \multirow{2}{*}{$\mathrm{C}$} & $\mathrm{C} 1$ & 0,3208 & 0,0118 & 3,6825 \\
\cline { 2 - 5 } & $\mathrm{C} 2$ & 0,4022 & 0,0128 & 3,1903 \\
\hline \multirow{2}{*}{$\mathrm{D}$} & $\mathrm{D} 1$ & 0,3217 & 0,0155 & 4,8248 \\
\cline { 2 - 5 } & $\mathrm{D} 2$ & 0,3246 & 0,0075 & 2,3260 \\
\hline
\end{tabular}

Cabe recordar que las muestras de cada lote fueron analizadas por triplicado y cada extracto obtenido también se analizó por triplicado en el UFLC, obteniendo para cada muestra nueve valores de concentración y un total de 72 datos para todas las muestras. Los valores promedio por muestra presentan una ligera variación, con coeficientes de variación menores al 10\% (entre 2,3260 y $8,2669 \%$ ), como se observa en la Tabla II.

En la Figura 2 se muestran los perfiles cromatográficos de la riboflavina extraída para el lote 1 de cada una de las cuatro marcas de arroz fortificadas y no fortificadas. El tiempo de retención (tR) del analito se presentó hacía los 1,97 minutos, tiempo de elución corto que hace al método muy rápido en cuanto al análisis cromatográfico, disminuyendo así el consumo de fase móvil.

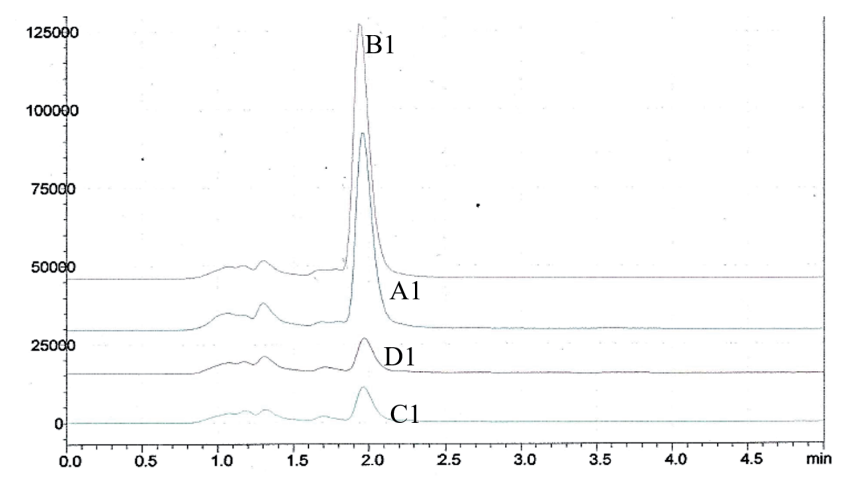

Figura 2. Cromatogramas de riboflavina obtenidos para las cuatro marcas de arroz. El tR de la riboflavina 1,97 min a longitud de excitación 450nm y de emisión 528nm.

Las muestras no fortificadas $(\mathrm{C} 1, \mathrm{C} 2$, D1 y D2) que fueron enriquecidas con $5,000 \mathrm{ppm}$ de riboflavina presentaron concentraciones de riboflavina de 4,631, 4,599, 4,659 y 4,656 $\mathrm{mg}$ de riboflavina/kg de arroz, respectivamente. Considerando el contenido natural encontrado según se reportó en la Tabla II, estos valores corresponden a respectivos porcentajes de recuperación de $92,62 \%, 91,98 \%, 93,18 \%$ y $93,12 \%$. 
Al comparar la concentración en ppm (mg de riboflavina/ $\mathrm{kg}$ de arroz) obtenida en el análisis de dos lotes de las muestras fortificadas (A1, A2, B1 y B2) y sin fortificar (C1, C2, D1 y D2), mediante el software $R$ versión 3.2.2., se obtuvo el diagrama de caja que se muestra en la Figura 3, (las líneas no identificadas en la Figura 3 corresponden a los lotes 2 de cada marca analizada, generada así por el software $R$ ).

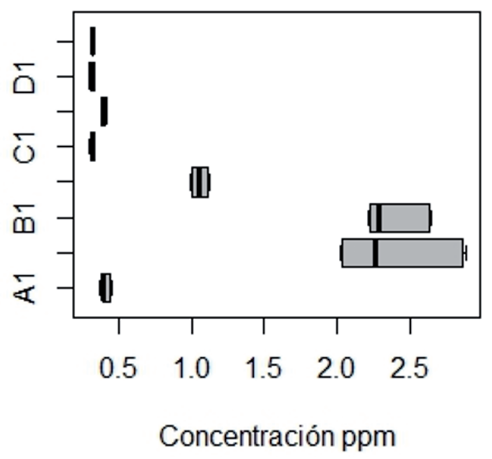

Figura 3. Comparación de la concentración de riboflavina ( $\mathrm{mg} / \mathrm{kg}$ de arroz) entre lotes de diferentes marcas de arroz fortificado y sin fortificar

Como se observa en la Figura 3, existe diferencia significativa en la concentración de riboflavina entre los lotes de todas las marcas, tanto fortificadas como no fortificadas $\left(p=2 \times 10^{-14}\right)$. Para las marcas que reportan fortificación, esta diferencia se observa entre lotes de la misma marca (A1 y A2) y (B1 y B2), así como entre los lotes de diferentes marcas (A y B). Respecto a las marcas que no reportan fortificación, los valores de la concentración de riboflavina reportados en la Tabla II son muy semejantes, con concentraciones que van de 0,3208 a 0,4022 ppm de riboflavina, que corresponde al contenido natural de riboflavina en el arroz, ya que los cereales son una fuente natural de ésta y otras vitaminas [7].

En el diagrama de caja mostrado en la figura 4, se compara la concentración de riboflavina entre los dos lotes de la marca fortificada B. Como se puede observar, se presenta una diferencia significativa entre las concentraciones de los lotes B1 y B2, con valores promedio de 2,374 y 1,055 mg de ribofla$\mathrm{vina} / \mathrm{kg}$ de arroz, respectivamente. Esta diferencia se corrobora con el valor de probabilidad de $1,41 \times 10^{-6}$. Respecto a las concentraciones de los lotes A1 y A2, con valores promedio de 0,4121 y 2,383 mg de riboflavina/ $\mathrm{kg}$ de arroz, respectivamente, mostrados en la Tabla II también se encuentra una diferencia bastante significativa $(\mathrm{p}=0.00139)$.

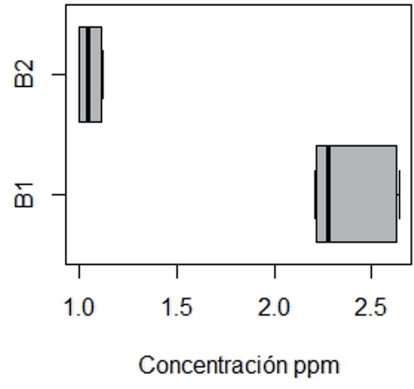

Figura 4. Comparación de la concentración de riboflavina ( $\mathrm{mg} / \mathrm{kg}$ de arroz) entre dos lotes de arroz de una misma marca fortificada.
Al comparar la concentración de riboflavina entre dos lotes de una misma marca de arroz no fortificado (D), mediante el diagrama de caja de la Figura 5, se observa que no se presenta diferencia significativa entre las concentraciones de los lotes D1 y D2, los valores promedio de la concentración son 0,3217 y $0,3246 \mathrm{mg}$ de riboflavina $/ \mathrm{kg}$ de arroz, respectivamente, verificado con un valor de $\mathrm{p}=0,787$. En la Tabla II se puede observar que las concentraciones promedio de los lotes $\mathrm{C} 1 \mathrm{y}$ C2 no fortificados, cuyos valores respectivos son $0,3208 \mathrm{y}$ $0,4022 \mathrm{mg}$ de riboflavina/ $\mathrm{kg}$ de arroz, presentan una diferencia significativa alta, según el valor de probabilidad de 0.00166 .

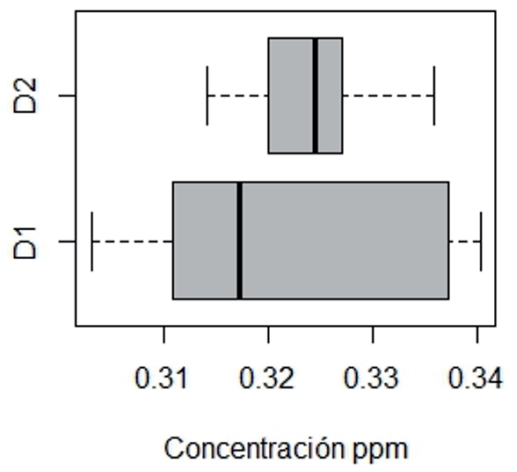

Figura 5. Comparación de la concentración de riboflavina ( $\mathrm{mg} / \mathrm{kg}$ de arroz) entre dos lotes de arroz de una misma marca no fortificada.

\section{Conclusiones}

Se estableció que para el análisis de riboflavina existe linealidad desde $2,500 \times 10^{-4}$ hasta $1,500 \mathrm{ppm}$, con un coeficiente de correlación de 0,99997 y al extrapolar a cero se mantiene el mismo coeficiente de correlación.

Entre diferentes lotes de arroz fortificado de la misma marca se presentan diferencias bastante significativas en el contenido de la riboflavina adicionada, lo que sugiere que las empresas que hacen la fortificación deben establecer un mecanismo que les permita llevar un mejor control en la calidad de su producto. En las muestras de arroz no fortificado, la concentración hallada corresponde al contenido natural de riboflavina en el arroz, con valores muy similares en todas las muestras.

Con el método estandarizado en este trabajo se obtienen porcentajes de recuperación de riboflavina alrededor del $93,00 \%$, que se debe tener en cuenta en el cálculo de la concentración de las muestras.

\section{Agradecimientos}

Las autoras expresan su agradecimiento al Fondo de Investigación Universitaria (FINU) de la Universidad Francisco de Paula Santander - Cúcuta, por la financiación de esta investigación (proyecto FINU 018-2017). 


\section{Referencias}

[1] M. E. Calles Porcel, "Estudio de un proceso de control analítico cuantitativo para la determinación de vitaminas hidrosolubles (tiamina, niacina, ácido fólico, riboflavina) por cromatografía liquida de alta eficiencia en harinas de trigo fortificadas". trabajo de fin de grado, Universidad de Guayaquil Facultad de Ciencias Químicas, Ecuador. 2004.

[2] J.F. Gregory III, Vitaminas. En: Química de los Alimentos. 2nd. ed. España. Acribia (Ed.). 2000.

[3] B. A. Fox and A. G. Cameron, Ciencia de los alimentos, nutrición y salud / B.A. Fox, A.G. Cameron, 2002.

[4] M. A. Royo Bordonada, coordinador, Nutrición en salud pública, Instituto de Salud Carlos III, Ministerio de Sanidad y Consumo, Madrid, 2007.

[5] N. Chen, S. Yin, X. Song, L. Fan \& H. Hu, "Vitamin B2 Sensitizes Cancer Cells to Vitamin-C-Induced Cell Death via Modulation of Akt and Bad Phosphorylation", Journal of agricultural and food chemistry, vol. 63, no.30, pp. 6739-6748, 2015.

[6] Organismo Andino de Salud, UNICEF. (2011, mayo). Respuestas de Salud y Nutrición Infantil Frente a la Crisis Económica en la Región Andina (1st ed.). [Online]. Disponible en: http://www.orasconhu.org/sites/default/files/files/LIBRO \%20RESPUESTAS\%20DE\%20SALUD\%20Y\%20NUT RICION\%20INFANTIL.pdf [Accedido en: nov. 10 de 2017]

[7] H. Pachón, "Fortificación del grano de arroz". Ministerio de Salud y Protección Social, Bogotá, Inf. téc. 2013.

[8] F. Tello Saavedra. et. al., "Estudio de pre-factibilidad para la instalación de una planta para obtener bioetanol a partir de residuos lignocelulósicos-cáscaras de arroz (Oriza sativa) en la Región Loreto". trabajo de fin de grado, Universidad Nacional de Amazonia Peruana, Perú. 2014.

[9] A. Gliszczynska-swiglo, A. Koziolowa, "Chromatographic determination of riboflavin and its derivatives in food", Journal of Chromatography, vol. 881, no. 1, pp 285-297, 2000.

[10] X. Tang, D. A. Cronin, and N. P. Brunton, "A simplified approach to the determination of thiamine and riboflavin in meats using reverse phase HPLC," Journal of Food Composition and Analysis, vol. 19, pp. 831-837, 2006.

[11] T. Fernández-Cárdenas, H. González-San Miguel, and L. Travieso, Determinación de vitaminas del Complejo B en Arthrospira máxima por cromatografía líquida de alta resolución vol. 42, 2017.

[12] L. A. Calderón Sánchez, O. Pazmiño Sánchez de Sayago,
"Estudio comparativo de las ventajas de la HPLC en relación con la Fluorometría en la determinación de vitaminas B1 y B2 en jarabes infantiles", Tesis Ph. D. Universidad de Guayaquil Facultad de Ciencias Químicas, Ecuador. 2004.

[13] L. Gil, et. al., "Evaluación del contenido de riboflavina por método de cromatografía líquida de alta resolución (HPLC) en harinas de lenteja (Lens e)", Alimentos Hoy, Revista de la Asociación Colombiana de Ciencia y Tecnología de Alimentos, vol. 22, no. 32, pp. 16 - 24, 2014.

[14] C. Bueno-Solano, et al., "Cuantificación de riboflavina (vitamina B2) en productos lácteos por HPLC", Revista Chilena de Nutrición, vol. 36, no. 2, pp. 136-142, 2009.

[15] M. G. Mencos De León. "Evaluación de dos métodos; cromatografía líquida de alta resolución (HPLC) y cromatografía líquida de ultra rendimiento (UPLC); para la cuantificación simultánea de cuatro vitaminas hidrosolubles en harina de trigo". Tesis. Facultad ciencias Químicas y Farmacia, Universidad de San Carlos de Guatemala. Guatemala. 2011.

[16] P. Wang, Y. Yin, S. A. Eremin, V. B. Rybakov, T. Zhang, $\mathrm{Z}$. $\mathrm{Xu}$, et al., "Indirect Competitive Immunoassay for Detection of Vitamin B2 in Foods and Pharmaceuticals," Journal of Agricultural and Food Chemistry, vol. 61, pp. 7048-7054, 2013.

[17] R Core Team. "R: A language and environment for statistical Computing". R Foundation for Statistical Computing. Vienna, Austria. Noviembre 2015. [Online]. Disponible en: https://www.R-project.org/ [Accessed: Jul-23-2018]

[18] Instituto de Salud Pública, "Determinación de riboflavina (vitamina B2) en harina de trigo. Método fluorométrico HPLC Basado en método 970.65 AOAC”, Ministerio de Salud - Gobierno de Chile, doc. tec. Versión 7. mayo 2016.

[19] J. C. Miller y J. N. Miller, Estadística para Química Analítica. 2nd. ed. Washington. Addison-Wesley Iberoamericana S. A. 1993. 